\title{
Pressões respiratórias em indivíduos submetidos a treinamento de endurance em membros inferiores e superiores
}

Jéssica Martins de Almeida Barbosa

Fisioterapeuta pelo Centro Universitário de Barra Mansa. jessicabarbosa2909@gmail.com

\section{Ana Carolina Ansaloni}

Fisioterapeuta pelo Centro Universitário de Barra Mansa. carol.ansaloni@hotmail.com

\section{Patricia Luciene da Costa Teixeira}

Professora do Curso de Fisioterapia do Centro Universitário de Barra Mansa. Mestre em Ciências Médicas pela Universidade Federal Fluminense. Especialista em Fisioterapia aplicada a terapia Intensiva Respiratória e Cardiovascular pela Universidade Salgado de Oliveira. Especialista em Acupuntura pelo Instituto Brasileiro de Estudos Homeopáticos. palufelix@uol.com.br 


\section{Resumo}

A perda progressiva de força muscular respiratória pode gerar alterações da caixa torácica e da coluna vertebral, consequentemente, podem desencadear modificações nos volumes e capacidades pulmonares. Este estudo experimental teve como objetivo avaliar as pressões respiratórias, por meio de um protocolo de exercícios em segmentos corporais diferentes. Um protocolo de endurance, soco em saco de pancadas, foi aplicado em dois grupos de 04 pacientes, sem doença respiratória conhecida, com idade média de 50,37 $\pm 18,02$ anos. Grupo 1 protocolo de em membros superiores, e grupo 2 protocolo em membros inferiores. $O$ treinamento de endurance quando realizado em segmentos corporais diferentes foi capaz de interferir de maneira significativa $(\mathrm{p}<0,05)$ na PImáx e $\mathrm{PFE} 1$, nos momentos pré e pós protocolo de endurance da amostra avaliada.

Palavras chave: Pressão inspiratória máxima. Pressão expiratória máxima. Fisioterapia.

\section{Abstract}

Progressive loss of respiratory muscle strength may cause changes in the rib cage and spine, consequently, may trigger changes in lung volume and capacity. This experimental study aimed to evaluate respiratory pressures through an exercise protocol in different body segments. An endurance protocol, punching bag punch, was applied to two groups of 04 patients without known respiratory disease with a mean age of $50.37 \pm 18.02$ years. Group 1 upper limb protocol and group 2 lower limb protocol. Endurance training, when performed in different body segments, was able to significantly interfere $(\mathrm{p}<0.05)$ in MIP and PEF1 before and after the endurance protocol of the evaluated sample.

Key words: Maximal inspiratory pressure; Maximal expiratory pressure. Physiotherapy. 


\section{Introdução}

As doenças respiratórias podem cursar com modificações estruturais do sistema respiratório que envolve os pulmões, a caixa torácica e a musculatura respiratória, especialmente o diafragma (JANSSENS et al., 1999); já as funcionais, incluem modificações nos volumes e capacidades pulmonares, decorrentes das alterações estruturais (ZAUGG et al., 2000).

Estresse emocional causa predominantemente respiração torácica, tensão muscular e aumento do trabalho respiratório em indivíduos saudáveis (DE TROYER et al., 1988). A persistência desses sintomas sem causas fisiológicas específicas são sugestivos de disfunção respiratória (DR) (COURTNEY et al., 2011). Os músculos acessórios da respiração também fornecem controle postural e facilitam a respiração durante situações estressantes, exercício, ou situações de perigo (JANSSENS et al., 2013). O estresse emocional e a ansiedade causam tensão nos músculos abdominais, o que inibe o movimento diafragmático, podendo induzir a respiração torácica, e maior recrutamento dos músculos acessórios da respiração (JONES et al., 2015), ocasionando dispneia, aumento do esforço muscular respiratório e fadiga muscular respiratória (VIDOTTO et al., 2019).

A Força Muscular Respiratória (FMR) vem sendo amplamente estudada e é mensurada através da Pressão Inspiratória máxima (PImáx) e da Pressão Expiratória máxima (PEmáx), além disso, são precisas, práticas e de simples aplicação (HOLM, 2004).

O exercício físico impõe demanda única e polivalente no sistema pulmonar; implícita nessa declaração está a ideia de que os meios fisiológicos desse sistema irão influenciar no desempenho do exercício, uma vez que as demandas metabólicas serão intensas a fim de manter a homeostasia (HOLM, 2004). O treinamento de endurance ou aeróbico baseia-se na realização de exercícios que hegemonicamente carecem de oxigênio para a produção de energia. Não obstante, são fundamentais para aprimorar as capacidades pulmonares e cardiovasculares.

Apesar de já terem sido extensamente estudados, os benefícios do treinamento da musculatura esquelética em pacientes portadores de pneumopatias crônicas, a realização deste estudo se justifica pelo fato de existir uma carência de informações na 
literatura nacional, acerca das modificações das pressões respiratórias, quando se treina separadamente membros inferiores e superiores.

Assim sendo, o presente estudo teve como objetivo avaliar os valores das pressões respiratórias, obtidas através de um protocolo de exercícios de endurance para Membros Superiores (MMSS) e Membros Inferiores (MMII) de pacientes traumato ortopédicos.

\section{Desenvolvimento}

\section{Metodologia}

Estudo transversal de natureza quantitativa, conduzido em uma Clínica Escola de Fisioterapia, situada no interior do estado do Rio de Janeiro após aprovação sob o $\mathrm{n}^{\circ}$ 1.053.587, pelo Comitê de Ética em Pesquisa (CEP) do Centro Universitário de Barra Mansa (UBM).

Foram incluídos 08 (oito) indivíduos sem doença respiratória conhecida, que aceitaram fazer parte do estudo, conforme o Termo de Consentimento Livre e Esclarecido (TCLE). Estes foram subdivididos em 02 (dois) grupos, sendo que o grupo 01 (G1) foi submetido a exercícios de endurance para membros superiores, e o grupo 02 (G2), exercícios de endurance para membros inferiores. Foram avaliados a pressão inspiratória e expiratória e o pico de fluxo expiratório no $1^{\mathrm{o}}$ segundo.

Foram incluídos neste estudo, pacientes com faixa etária superior a 18 anos, de ambos os gêneros, que estivessem tratando de problemas posturais tais como escoliose, cifose ou lordose, sem doença pulmonar conhecida.

Foram excluídos pacientes portadores de qualquer qualidade de demência, afasia sensorial, que apresentasse déficit auditivo, visual e/ou motor importante, ou qualquer tipo de deformidade ou distúrbio cinético funcional, que causassem prejuízo na biomecânica dos grupos musculares envolvidos, e/ou com histórico patológico pregresso que envolvesse o sistema respiratório.

Antes e após a realização do protocolo de exercício, todos os participantes passaram por avaliação da pressão arterial (PA), através da técnica auscultatória realizada no braço esquerdo empregando-se esfigmomanômetro; frequência de pulso (FP) e 
saturação parcial de oxigênio ( $\mathrm{SpO} 2)$, através de oxímetro de pulso digital; avaliação visual da frequência respiratória (FR); avaliação do pico de fluxo expiratório no $1^{\mathrm{o}}$ segundo (PFE1) por peakflow meter, das pressões inspiratórias máximas (PImáx) e pressão expiratória máxima (PEmáx), através da manovacuometria, e medidas antropométricas, incluindo medidas de peso e altura por balança, e circunferências da cintura e quadril e cirtometria torácica, por fita métrica milimetrada.

Passaram também por avaliação de 1Repetição Máxima (1RM), que consiste na quantidade máxima de peso levantada uma vez, durante a realização de um exercício padronizado de levantamento de peso, respeitando os critérios da fase concêntrica e excêntrica. A capacidade de 1RM foi determinada na presença de exaustão voluntária, e/ou através da incapacidade de manter o padrão do movimento. Para o teste 1RM de um grupo muscular, é escolhido um peso, ao dispositivo do exercício, porém abaixo da capacidade máxima de levantamento do indivíduo. Se uma repetição é completada, acrescenta-se peso ao dispositivo do exercício, até alcançar a capacidade máxima de levantamento. Os acréscimos de peso foram de $0,5 \mathrm{Kg}$ durante o período de avaliação. Essa técnica foi utilizada com halteres para MMSS e caneleiras para MMII, e apenas serviu como referência para certificação de que as cargas escolhidas para os grupos não estavam acima do limite mínimo individual. A carga registrada como 1-RM é aquela na qual cada indivíduo consegue completar uma única repetição máxima (CLARKE, 1973 apud GURJÃO et al., 2005).

Após este procedimento padrão os participantes aguardavam 10 minutos para iniciar o protocolo de exercício. Os pacientes do G1 ficaram de pé e realizaram 10 minutos de aquecimento em MMSS, dando socos no ar, seguindo sinais sonoros a cada segundo. Após este procedimento, fizeram 3 séries de 5 repetições de exercícios de flexoextensão de cotovelos, mantendo-os na linha média do corpo por 5 segundos, abdução e flexão de ombros mantendo-os no plano dos ombros por 5 segundos, sendo que os exercícios foram realizados com a carga de $1 \mathrm{~kg}$ para as mulheres e $2 \mathrm{~kg}$ para os homens, sem acréscimo de carga posteriormente. Antes de passarem para as outras posturas durante 10 minutos, voltaram a dar socos no ar, obedecendo a cadência sonora a cada segundo, em decúbito ventral, seguindo com 3 séries de 5 repetições de extensão de ombros, mantendo por 5 segundos. Ao final, foram submetidos à massoterapia para relaxamento da musculatura envolvida. 
Os participantes do G2 ficaram sentados em uma cadeira confortável o suficiente para que não houvesse desconforto postural e fizeram 10 minutos de aquecimento em MMII, dando pontapés no ar, seguindo sinais sonoros a cada segundo; após este procedimento, fizeram 3 séries de 5 repetições de exercícios de extensão de joelhos, com a carga de $1 \mathrm{~kg}$ para todos os participantes, sem acréscimos de carga posteriormente. Antes de passarem para as outras posturas, durante 10 minutos, voltaram a dar pontapés no ar, obedecendo a cadência sonora a cada segundo; em decúbito lateral, fizeram 3 séries de 5 repetições de abdução e adução de coxofemoral, e, em decúbito ventral, 3 séries de 5 repetições de flexoextensão de joelhos. Ao final, foram submetidas à massoterapia para relaxamento da musculatura envolvida.

Para definir a carga a ser utilizada, a pesquisa baseou-se nos parâmetros do teste de $1 \mathrm{RM}$.

Após a triagem, os participantes foram submetidos a uma anamnese/exame fisiofuncional, antes das sessões, objetivando detectar possíveis contraindicações para realização das técnicas de análise. Passaram também por avaliação da Pressão Arterial (PA), através da técnica auscultatória realizada no braço esquerdo, meramente por padronização da técnica, empregando-se esfigmomanômetro analógico; Frequência de Pulso (FP); avaliação visual da Frequência Respiratória (FR) e Saturação parcial de Oxigênio (SpO2) por oxímetro digital; medidas antropométricas, incluindo medidas de peso e altura respectivamente por balança e estadiômetro; circunferência da cintura, do quadril e, cirtometria torácica por fita métrica milimetrada não extensível; avaliação do pico de fluxo expiratório no $1^{\mathrm{o}}$ segundo por peakflow meter, da pressão inspiratória máxima (PImáx.) e pressão expiratória máxima (PEmáx.), através da manovacuometria, registrando-se o melhor valor após três mensurações. Em todas as avaliações, os participantes permaneceram em sedestação, com dorso apoiado na cadeira e pés apoiados no chão.

Quando identificada alguma alteração ligada aos sistemas cardiorrespiratórios, vascular e/ou musculoesquelético, o participante foi comunicado e recebeu encaminhamento para profissional especializado. Do contrário, dava-se continuidade ao protocolo de pesquisa, com reavaliações antes e após cada sessão.

Pelo fato dos participantes não possuírem experiência prévia em exercícios com pesos, foi adotado um processo de familiarização prévia aos gestos exigidos nos 
exercícios. Para avaliar a carga a ser imposta, o voluntário realizou três séries de 10-12 repetições de um exercício, com a carga progressiva de 0,5 kg, $1 \mathrm{~kg}$ e $2 \mathrm{~kg}$ (MMSS) e 0,5 kg, $1 \mathrm{~kg}$ e 1,5 (para MMII). O intervalo de recuperação entre as séries e entre exercícios foi de aproximadamente 90 segundos; o exercício foi realizado em uma tentativa de repetição máxima para cada carga. Cada um dos dois exercícios foi precedido por uma série de aquecimento (6 a 10 repetições), com a carga mínima. Após dois minutos de repouso, os testes eram iniciados.

\section{Análise dos dados}

Após a coleta, os dados individuais foram analisados separadamente por meio do Software Microsoft Excel para Windows e GraphPadPrism. Dados das características clínicas foram expressos por média e desvio Padrão (DP), para variáveis contínuas e valores absolutos, ou percentuais para variáveis categóricas. Foi utilizado Teste $\mathrm{t}$ pareado, para comparação dos momentos pré e pós-treinamento em cada grupo, e Mann-Whitney para comparação entre os grupos.

\section{Resultados e discussão}

A amostra de conveniência foi constituída por 08 indivíduos de ambos os sexos, sendo 06 mulheres e 02 homens, com idade média de 50,37 $\pm 18,02$ anos.

Os participantes foram divididos em 02 grupos de 4 participantes cada, sendo o que o G1 realizou protocolo de endurance em MMSS e o G2 protocolo para MMII.

As características antropométricas dos grupos estão apresentadas na tabela 1.

Tabela 1 - Dados antropométricos da amostra avaliada. Valores expressos em mínimo, máximo, média \pm DP da média. 
BARBOSA, J.M.A.; ANSALONI, A.C.; TEIXEIRA, P.L.C. Pressões respiratórias em indivíduos submetidos a treinamento de endurance em membros inferiores e superiores. R. Cientifica UBM - Barra Mansa (RJ), ano XXIX, v. 21, n. 41, 2. Sem. 2019. p. 27-40.

ISSN 1516-4071

G1_MMSS (n=4) Mínimo Máximo Média DP

\begin{tabular}{llll}
\hline Peso $(\mathrm{Kg})$ & 53,2 & 77,8 & $67,3 \pm 12,68$ \\
Altura $(\mathrm{cm})$ & 143 & 173 & $163,5 \pm 14,01$ \\
Cintura $(\mathrm{cm})$ & 79 & 91 & $86,75 \pm 5,43$ \\
Quadril $(\mathrm{cm})$ & 93 & 104 & $97,75 \pm 5,61$ \\
Perimetria torácica & 97 & 109 & $100,75 \pm 5,56$
\end{tabular}

G2_MMII (n=4)

\begin{tabular}{llll} 
Peso $(\mathrm{Kg})$ & 53,2 & 62,8 & $58 \pm 4,08$ \\
\hline Altura $(\mathrm{cm})$ & 16 & 163 & $120,75 \pm 61,17$ \\
Cintura $(\mathrm{cm})$ & 85 & 109 & $95,75 \pm 10,77$ \\
Quadril $(\mathrm{cm})$ & 93 & 113 & $99,75 \pm 8,84$ \\
Perimetria torácica & 95 & 115 & $101,5 \pm 8,81$ \\
\hline
\end{tabular}

$\mathrm{Na}$ tabela a seguir estão apresentados os valores médios \pm desvio padrão $( \pm \mathrm{DP})$, variações mínimas e máximas, bem como a significância estatística $\mathrm{p}<0,005$ das variáveis fisiológicas avaliadas (Teste t pareado), mostrando que quando comparamos os momentos pré e pós-treinamento de endurance em MMSS, apenas a FR e a PAD apresentou $\mathrm{p}<0,05$.

Tabela 2: Valores médios $\pm \mathrm{DP}$, variação mínimo - máximo e valor de $\mathrm{p}$ das variáveis fisiológicas avaliadas, dos grupos submetido ao treinamento em MMSS e MMII 


\begin{tabular}{|c|c|c|c|c|c|}
\hline Variáveis & \multicolumn{2}{|l|}{ Pré } & \multicolumn{3}{|l|}{ Pós } \\
\hline MMSS & & & & & \\
\hline & Média \pm DP & $\begin{array}{l}\text { Variação } \\
\text { (Mín - Máx) }\end{array}$ & Média $\pm D P$ & $\begin{array}{l}\text { Variação } \\
\text { (Mín - Máx) }\end{array}$ & $P$ \\
\hline FR & $20,8 \pm 3,10$ & $18-25$ & $22,8 \pm 3,7$ & $19-28$ & $N s$ \\
\hline $\mathrm{FP}$ & $77,3 \pm 9,88$ & $65-89$ & $77,3 \pm 10,72$ & $67-87$ & 0,0405 \\
\hline PAS & $\begin{array}{l}117,8 \quad \pm \\
13,67\end{array}$ & $105-136$ & $127,0 \pm 7,87$ & $118-137$ & $N s$ \\
\hline PAD & $70,3 \pm 4,03$ & $67-76$ & $75,0 \pm 6,16$ & $70-84$ & $N s$ \\
\hline $\mathrm{SpO}_{2}$ & $97,5 \pm 0,58$ & $97-98$ & $97,3 \pm 0,5$ & $97-98$ & 0,0409 \\
\hline PImáx & $\begin{array}{l}106,3 \quad \pm \\
44,4\end{array}$ & $40-132$ & $111,0 \pm 25,7$ & $75-136$ & Ns \\
\hline PEmáx & $70,5 \pm 37,9$ & $38-115$ & $71,3 \pm 30,47$ & $40-103$ & $N s$ \\
\hline P. Flow & $378 \pm 58,8$ & $286-444$ & $231,5 \pm 47,29$ & $308-450$ & $N s$ \\
\hline MMII & & & & & \\
\hline FR & $22 \pm 3,16$ & $19-26$ & $25,5 \pm 4,04$ & $22-31$ & $n s$ \\
\hline FP & $73 \pm 16,83$ & 57-96 & $75,8 \pm 16,13$ & $62-98$ & 0,0405 \\
\hline PAS & $\begin{array}{l}117,5 \quad \pm \\
7,77\end{array}$ & $106-123$ & $134,3 \pm 2,87$ & $130-136$ & $n s$ \\
\hline PAD & $74,5 \pm 1,29$ & $73-76$ & $74,5 \pm 4,36$ & $71-80$ & $n s$ \\
\hline $\mathrm{SpO}_{2}$ & $96,8 \pm 0,5$ & $96-97$ & $97,0 \pm-$ & $97-97$ & 0,0409 \\
\hline PImáx & $\begin{array}{l}46,8 \quad \pm \\
19,74\end{array}$ & $21-69$ & $51,5 \pm 20,66$ & $27-75$ & $n s$ \\
\hline PEmáx & $53,3 \pm 8,10$ & $44-61$ & $52,8 \pm 10,36$ & $39-64$ & $n s$ \\
\hline P. Flow & $\begin{array}{l}239,5 \quad \pm \\
9,98\end{array}$ & $226-248$ & $231,5 \pm 47,29$ & $189-286$ & $n s$ \\
\hline
\end{tabular}

Valores expressos em média $\pm \mathrm{DP}$ onde: $\mathrm{FR}=$ frequência respiratória; $\mathrm{FP}=$ frequência de pulso; $\mathrm{PAS}=$ pressão arterial sistólica; $\mathrm{PAD}=$ pressão arterial diastólica; $\mathrm{SpO}_{2}=$ saturação parcial de oxigênio; PImáx. = pressão inspiratória máxima; PEmáx. = pressão expiratória máxima; $\mathrm{PFE1} \mathrm{=} \mathrm{pico} \mathrm{de} \mathrm{fluxo} \mathrm{expiratório} \mathrm{no}$ $1^{\text {o }}$ segundo. 
A fraqueza muscular respiratória é um importante problema clínico. A disponibilidade de vários testes da função muscular respiratória aumenta a precisão do diagnóstico e possibilita a avaliação em várias circunstâncias clínicas (STEIER, 2007). Do mesmo modo, esses testes também são utilizados para confirmação e acompanhamento de muitos procedimentos técnicos empregados na fisioterapia respiratória, principalmente em asmáticos (BRIGHAM, 2015).

A avaliação da força muscular respiratória é uma técnica não invasiva e de baixo custo realizada por equipamentos portáteis de fácil manuseio (CAVALCANTE, 2010). Quando há diminuição da PImáx com PEmáx normal, pensa-se em desvantagem mecânica (MONTEIRO et al., 2003). Entretanto, o uso de testes únicos como PImax, PEmax e/ou outros testes individuais disponíveis para força muscular inspiratória, diafragma e expiratória tendem a superdiagnosticar fraqueza. Já as combinações dos testes aumentam a precisão diagnóstica (STEIER, 2007)

A medida do fluxo expiratório é uma forma de se avaliar a função pulmonar, permitindo alertar sobre a diminuição da função respiratória (BARNES, 1985 apud FRITSCHER, 2007). A queda nos valores do Pico de Fluxo Expiratório, antes da manifestação de sintomas patológicos do sistema respiratório, indica que deve ser iniciar um tratamento profilático ou curativo (GIANNINI et al., 1997)

Já foi demonstrado que o padrão respiratório pode ser alterado com a posição do corpo (MACHADO, 2007).

Neste estudo, durante as mensurações do G2, não foi observada uma variação tão expressiva das pressões respiratórias bem como no PFE1, talvez esse resultado possa ter sido influenciado por outliers relacionado à obesidade dentro do grupo. Já foi descrito na literatura que acúmulo de tecido adiposo traz agravos específicos à saúde dos indivíduos, e leva à diminuição do desempenho físico (STEIN; COLDITZ, 2004; PINHEIRO et al, 2004). Já os dados relativos às pressões respiratórias e o pico de fluxo expiratório no primeiro segundo, obtidos pelos diferentes protocolos utilizados pelos grupos G1 e G2, também sofrem interferência, independentemente da posição corporal adotada pelos participantes.

A figura 1 apresenta o comportamento do PFE1 nos momentos pré e póstreinamento, com diferença significativa antes do treinamento de endurance entre os 
grupos, diferentemente da pressão PEmáx., que não apresentou diferença estatisticamente significativa, dados não apresentados.

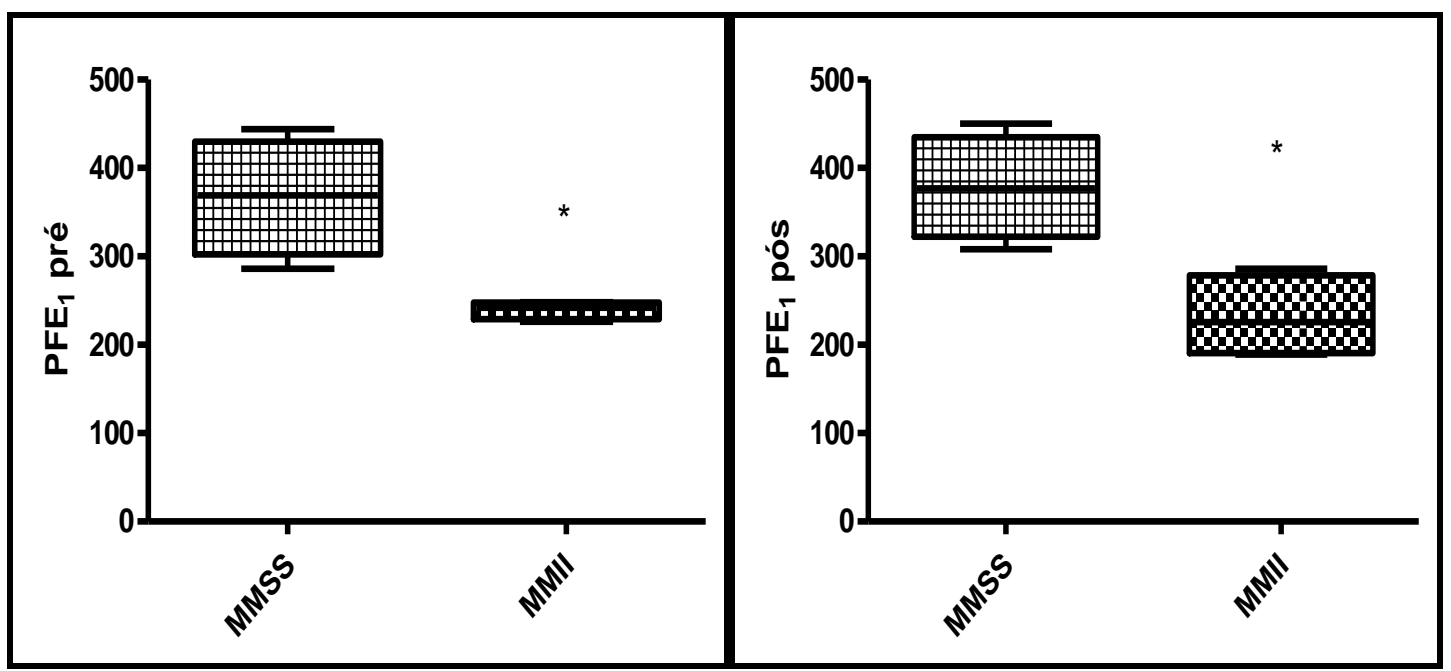

Fig. 1: Comparação do comportamento do $\mathrm{PFE}_{1}$ pré e pós-treinamento em ambos os grupos $\mathrm{p}=0,02$. Fonte: Dados do estudo.

Em relação ao comportamento das pressões pós-treinamento de endurance, apenas a PEmáx não apresentou significância estatística, entre os grupos submetidos ao treinamento de MMSS e MMII. As variáveis PImáx apresentaram p = 0,04, como demonstrado na figura a seguir.

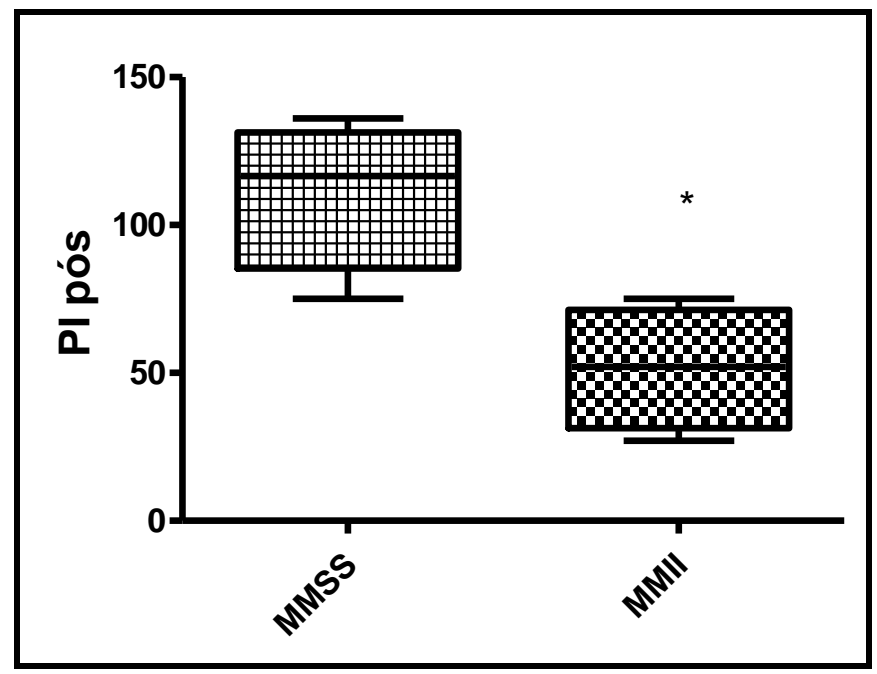

Fig. 2: Comparação do comportamento do PI máx. pós-treinamento em ambos os grupos p=0,04. Fonte: Dados do estudo. 


\section{Considerações Finais}

As pressões respiratórias sofreram interferência do treinamento realizado em segmentos corporais diferentes. Apesar de não terem sido encontradas diferenças estatisticamente significativas entre os momentos pré e pós-treinamento de endurance entre os grupos G1 e G2, ambas modalidades de treinamento foram capazes de interferir de forma significativa no PFE1 nos dois momentos. Não obstante, importante ressaltar que o tamanho amostral pequeno foi uma limitação potencial, cabendo, portanto, realização de novos estudos, incluindo um número maior de sujeitos para confirmar se os resultados obtidos não ocorreram ao acaso.

\section{Referências}

Cavalcante Marcelino A.M ; Justino da Silva H. Papel da pressão inspiratória máxima na avaliação da força muscular respiratória em asmáticos Revisão sistemática. Rev Port Pneumol. v. 16 n. 3 p 463-70, 2010.

COURTNEY, R. et al. Medically unexplained dyspnea: partly moderated by dysfunctional (thoracic dominant) breathing pattern. J Asthma v. 48 n. 3 p 259-65, 2011. DOI: https://doi.org/10.3109/02770903.2011.554942

DE TROYER, A.; ESTENNE, M. Functional anatomy of the respiratory muscles. Clin Chest Med. v. 9 n. 2 p. 175-93, 1988.

EMILY P. et al. Diagnosis of asthma: diagnostic testing West, MD, MHS International Forum of Allergy \& Rhinology, v. 5, n. S1, 2015 
FRITSCHER, C.C.; KAHAN, F.; ZETLER, E. Um guia para orientação de pacientes asmáticos. Sociedade Brasileira de Pneumologia e Tisiologia. Disponível em: http://www.sbpt.org.br/ rsp. Acesso em: 20 de março de 2007.

GIANNINI, D.; PAGGIARO, P.L. et al. Comparação entre pico de fluxo expiratório e volume expiratório forçado no primeiro segundo (VEF1) durante broncoconstrição induzida por diferentes estímulos. J Asma, v 34 n. 2: p 105-11, 1997.

GURJÃO, A. L. et al. Variação da força muscular em testes repetitivos de 1-RM em crianças pré-púberes. Revista Brasileira de Medicina do Esporte, v.11, n.6, nov./dez. 2005.

HOLM, P. et al. Endurance training of respiratory muscles improves cycling performance in fit young cyclists. BioMed Central Physiology, v.6, n. 4. 2004.

JANSSENS, J. P.; PACGE, J. C.; NICOD, L. P. Physiological changes in respiratory function associated with aging. European Respiratory Society, v.13, n.1, p.197-205, 1999.

JANSSENS, L. et al. Postural strategy and back muscle oxygenation during inspiratory muscle loading. Med Sci Sports Exerc. 2013;45(7):1355-62.

DOI: https://doi.org/10.1249/MSS.0b013e3182853d27

JONES, M. et al. Does manual therapy provide additional benefit to breathing retraining in the management of dysfunctional breathing? A randomised controlled trial. Disabil Rehabil. 2015; 37(9):763-70.

DOI: $\underline{\text { https://doi.org/10.3109/09638288.2014.941020 }}$

MACHADO, M. G. R. Livro:Bases da Fisioterapia Respiratória:Terapia Intensiva e Reabilitação. Guanabara Koogan, 2007. 


\section{MONTEIRO, J.G.B.; FERNANDES, L.C.; MACHADO, O. Atuação}

Fisioterapêutica no Comprometimento Cardiorrespiratório na Síndrome de Guillain-

Barré: relato de caso. 2003. 25 f. Monografia (obtenção do título de aprimoramento em Fisioterapia

Respiratória em Terapia Intensiva) Hospital Nossa Senhora da Penha, São Paulo: 2003.

STEIN, C. J.; COLDITZ, A.G. The epidemic of Obesit. The Journal of Clinical and Metabolism, June, 2004.

STEIER J ${ }^{1}$, KAUL S. et al. O valor de vários testes de força muscular respiratória. Tórax. v. 62, n. 11, p. 975-80, 2007

VIDOTTO, L. S. et al . Disfunção respiratória: o que sabemos? J. bras. pneumol., São Paulo, v. 45, n. 1, e20170347, 2019. DOI: http://dx.doi.org/10.1590/18063713/e20170347.

ZAUGG, M.; LUCCHINETTI, E. Respiratory function in the elderly. Anesthesiol Clin North America, v.18, n.1, p.47-58, 2000. 